\title{
Acordo Mercosul - União Europeia: Os Perigos para a Saúde Pública Brasileira
}

\author{
Bianca Carvalho Pontes ${ }^{*}$
}

\section{Resumo:}

Este artigo tem como objetivo analisar como as medidas de proteção à propriedade intelectual impactam a saúde pública e como as medidas TRIPS Plus sugeridas pela União Europeia no Acordo de Livre Comércio entre o Mercosul e a União Europeia afetam negativamente o programa de assistência farmacêutica do Sistema Único de Saúde. O direito à saúde no Brasil está assegurado pela Constituição Federal de 1988, desse modo, o direito à saúde é um direito constitucional que deve ser assegurado e protegido pelo Estado brasileiro. Portanto, é importante debater e entender como o Acordo de Livre Comércio entre o Mercosul e a União Europeia afeta os programas de assistência farmacêutica do Sistema Único de Saúde, com o objetivo de defender a saúde pública brasileira. A União Europeia possui um vasto histórico de implementar de maneira sutil medidas TRIPS Plus nos textos dos Acordos bilaterais que negocia com outros países e blocos econômicos mais fracos política e economicamente, pois estes necessitam do suporte do bloco europeu para fortalecerem suas economias. Desse modo, ficam reféns das exigências feitas pela União Europeia no capítulo de propriedade intelectual dos Acordos. O artigo conclui que caso seja elevado o nível de proteção da propriedade intelectual no Brasil como deixada em aberto pela União Europeia no texto final do Acordo de Livre Comércio entre os dois blocos, o programa nacional de assistência farmacêutica será afetado negativamente e os custos do Ministério da Saúde para a compra de medicamentos patenteados aumentará significativamente.

Palavras-chave: Propriedade Intelectual; TRIPS PLUS; Sistema Único de Saúde; Acordo de Livre Comércio; Medicamentos Genéricos.

\begin{abstract}
:
This article aims to analyze how intellectual property protection measures impact Public Health and how the TRIPS Plus measures suggested by the European Union in the Free Trade Agreement between Mercosur and the European Union negatively affect the pharmaceutical assistance program of the Brazilian National Health System. The Federal Constitution of 1988 assures the right to health in Brazil, thus, the right to health is a constitutional right. Therefore, it is important to discuss and understand how the Free Trade Agreement between Mercosur and the European Union affects the pharmaceutical assistance programs of the Unified Health System, with the objective of defending Brazilian public health. The European Union is known for subtly implementing TRIPS Plus measures in bilateral agreements texts when negotiating. Especially regarding countries that are weaker politically and economically. It takes advantage because most of them need European support to strengthen their economies and, therefore, they do not impose any demands against the European Union when discussing intellectual property agreements.. The article concludes that if the level of intellectual property protection in Brazil is raised as left open by the European Union in the final text of the Free Trade Agreement between the two blocks, the

\footnotetext{
* Graduação em andamento em Relações Internacionais pela PUC-Rio, com domínio adicional em Gestão e Avaliação de Políticas Públicas. Bolsista de Iniciação Científica pelo Programa de Educação Tutorial (PET) do IRI/PUC-Rio. Email: carvalho.biancacp@gmail.com.
} 
national pharmaceutical assistance program will be negatively affected and the Ministry of Health's costs for the purchase of patented medicines will increase significantly.

Keywords: Intellectual Property; TRIPS PLUS; Unified Health System; Free Trade Agreement; Generic Drugs.

\section{Introdução}

Depois de mais de dez anos de negociações, o Acordo de Livre Comércio entre o Mercosul e a União Europeia foi assinado em 2019 No entanto, apesar das comemorações, o capítulo de propriedade intelectual do acordo representa um desafio os moldes da saúde pública dos países membros do Mercosul, especialmente para o Brasil.

O Sistema Único de Saúde (SUS), criado pela Constituição Federal de 1988, determina que a saúde é um direito de todos e dever do Estado. De acordo com o artigo $7^{\circ}$ da Lei $\mathrm{N}^{\mathrm{o}} 8.080 / 90$, o SUS tem como princípios:

I) Equidade: tem como objetivo diminuir as desigualdades, deste modo, investe-se mais onde a carência é maior para que todos tenham as mesmas condições de acesso à saúde; II) Integralidade: é o conjunto articulado e contínuo das ações dos serviços de reabilitação, serviços preventivos e curativos individuais e coletivos, exigidos para cada caso em todos os níveis de complexidade do sistema; e III) Universalidade: garante que qualquer cidadão tenha direito de acesso a todos os serviços públicos de saúde, em todos os níveis de assistência. (BRASIL, Artigo $7^{\circ}$ da lei $n^{\circ} 8.080 / 1990$, grifo nosso).

Além disso, conforme consta no Artigo $16^{\circ}$ da lei $\mathrm{n}^{\mathrm{o}} 8.080 / 1990$, no campo de atuação do SUS está incluída a execução de ações de vigilância sanitária; o controle e a fiscalização de serviços, produtos e substâncias de interesse para a saúde; a físcalização e a inspeção de alimentos, água e bebidas para consumo humano. Desse modo, todos utilizam o SUS direta ou indiretamente, visto que desde o alimento que é consumido por cada brasileiro até a cirurgia que é realizada em um hospital público está sob responsabilidade do SUS.

A pesquisa irá abordar os impactos do acordo entre a União Europeia e Mercosul na saúde pública brasileira, visto que o Sistema Único de Saúde é o único sistema público de saúde do mundo que atende mais de 100 milhões de habitantes, garantindo o acesso universal e gratuito para todos os cidadãos brasileiros.

Ademais, o Sistema Único de Saúde está assegurado pela Constituição Federal de 1988, o que torna o direito à saúde no Brasil um direito constitucional, sendo o Estado responsável por assegurar a saúde pública para todos os brasileiros. Além disso, o SUS renova o conceito de saúde no Brasil pois não atua apenas como um sistema de saúde curativo, mas é também um sistema de saúde preventivo e de tratamento. Dito isto, para que seja possível entender como as medidas TRIPS Plus sugeridas pela União Europeia no capítulo de propriedade intelectual do acordo de livre comércio com o Mercosul 
afetam o programa nacional de assistência farmacêutica, o artigo será divido em quatro seções com o objetivo de responder à pergunta: "Como o acordo entre o Mercosul e a União Europeia impacta o programa de assistência farmacêutica do Sistema Único de Saúde?".

Ainda é importante destacar que, desde a assinatura do Agreement on TradeRelated Aspects of Intellectual Property Rights (Acordo TRIPS) pelos países membros da Organização Mundial do Comércio (OMC) em 1995, a saúde pública dos países em desenvolvimento tem sofrido com diversas restrições a produção de medicamentos genéricos.

Os medicamentos genéricos são essenciais para a manutenção da Política Nacional de Medicamentos do Brasil, desse modo, ao longo do artigo será analisado como as medidas de proteção à propriedade intelectual impactam a saúde pública brasileira e como as medidas TRIPS Plus sugeridas pela União Europeia no Acordo de Livre Comércio entre o Mercosul e a União Europeia afeta negativamente o programa de assistência farmacêutica do Sistema Único de Saúde.

Vale destacar que o direito à saúde e o direito de acesso aos medicamentos além de estarem assegurados pela Constituição Federal de 1988 também encontram claro respaldo em outros documentos internacionais, como: na Carta das Nações Unidas (1945); na Declaração Universal dos Direitos Humanos (1948) e na Constituição da Organização Mundial da Saúde (1948). O direito de propriedade intelectual possui impacto direto no direito à saúde, pois as patentes farmacêuticas encarecem os medicamentos através da monopolização de seu preço.

Embora o acordo TRIPS garanta flexibilidades que podem ser adotadas pelos países a fím de protegerem a saúde pública nacional, essas salvaguardas se mostram insuficientes na garantia do direito de acesso à saúde e, consequentemente, o direito de acesso a medicamentos. Isso acontece porque há um conflito de interesses, ou seja, os países do Norte Global criam entraves com o intuito de impedir que os países do Sul Global façam uso de tais flexibilidades com o objetivo de defenderem os interesses econômicos de suas indústrias farmacêuticas.

Portanto, fica nítido que desenvolvimento e saúde são dois temas interligados visto que um impacta diretamente o outro. Quando olhamos para o acordo TRIPS essa relação entre desenvolvimento e saúde fica nítida, posto que o acordo estabeleceu padrões mínimos de proteção da propriedade intelectual e colocou como obrigatória que todos os países membros da $\mathrm{OMC}$ reconhecessem patentes para todos os campos tecnológicos com o intuito de assegurar o desenvolvimento em todos os campos tecnológicos, como a área farmacêutica.

Além disso, o estabelecimento de cláusulas TRIPS Plus nos acordos bilaterais de livre comércio configura uma ameaça ainda maior à saúde pública, visto que tais cláusulas elevam o nível de proteção da propriedade intelectual e dificultam o uso das flexibilidades asseguradas pelo Acordo TRIPS. O acordo entre o MERCOSUL e a União Europeia passou por negociações que envolviam o estabelecimento de cláusulas TRIPS Plus no capítulo de propriedade intelectual que poderiam afetar fortemente a saúde pública brasileira. Entretanto, mesmo com a retirada das cláusulas explícitas de medidas TRIPS 
Plus o texto final do acordo deixou aberto um espaço para uma possível elevação do nível de proteção da propriedade intelectual nos países membros do Mercosul.

Para tanto, foi utilizada na metodologia da pesquisa a entrevista ${ }^{1}$ concedida pela pesquisadora em saúde pública da Escola Nacional de Saúde Pública Sérgio Arouca da Fundação Oswaldo Cruz (ENSP/FIOCRUZ) Gabriela Chaves sobre o acordo entre o Mercosul e a União Europeia para a autora do presente artigo. Com o objetivo de analisar o impacto do Acordo TRIPS na produção de medicamentos genéricos e o impacto das medidas TRIPS Plus sugeridas pela União Europeia, também foi utilizado no artigo fontes primárias (texto do capítulo de propriedade intelectual do acordo de livre comércio entre o Mercosul e a União Europeia, Lei No 8080/90; Lei No 9.279/96; Lei No 9.787/99, portaria $\mathrm{N}^{0} 3.916 / 98$, texto do acordo TRIPS estabelecido pela Organização Mundial do Comércio e o texto da Declaração de Doha sobre o Acordo TRIPS e a saúde pública de 2001).

Na primeira seção será apresentado o que é o Acordo sobre Aspectos dos Direitos de Propriedade Intelectual Relacionados ao Comércio (em inglês: Agreement on TradeRelated Aspects of Intellectual Property Rights; TRIPS) e como tal acordo impactou a saúde pública dos países membros da Organização Mundial do Comércio, em especial os países em desenvolvimento. Em seguida, irei abordar como foi criado o programa de assistência farmacêutica do Sistema Único de Saúde e a importância dos medicamentos genéricos para a manutenção e sustentabilidade deste programa garantindo, assim, que todos os cidadãos brasileiros tenham acesso de forma gratuita ao tratamento medicamentoso do qual necessitam.

Já na terceira seção será analisado o capítulo de propriedade intelectual do acordo de livre comércio entre o Mercosul e a União Europeia, com o objetivo de entender como as medidas TRIPS Plus sugeridas pela União Europeia são prejudiciais para a saúde pública brasileira, dando ênfase nas consequências dessas medidas para o programa de assistência farmacêutica do Sistema Único de Saúde. Por fim, serão expostas as conclusões sobre o possível impacto das medidas TRIPS Plus sugeridas pela União Europeia no Sistema Único de Saúde.

\section{O Acordo TRIPS}

Durante a década de 70, os países asiáticos de industrialização recente começaram a lançar, baseado em cópia, algumas tecnologias e passaram a disputar o mercado. Com isso, como pontua a pesquisa "TRIPS e a saúde pública: Implicações e perspectivas" (DE MELLO E SOUZA, 2011), começou a surgir uma pressão para incluir o tema de direitos de propriedade intelectual dentro da agenda do General Agreement on Tariffs and Trade (GATT), vinda principalmente das indústrias norte americanas de software, microeletrônica, entretenimento, química, farmacêutica e de biotecnologia, que alegavam estar sofrendo perdas em terceiros mercados devido à ausência de uma proteção adequada

\footnotetext{
${ }^{1}$ Entrevista realizada pela autora do artigo sobre patentes farmacêuticas; assistência farmacêutica no SUS e o capítulo de propriedade intelectual do Acordo entre o Mercosul e a União Europeia no dia 25 de novembro de 2020 .
} 
à propriedade intelectual.

Como afirma o estudo "Tratado de Livre Comércio União Europeia- Mercosul: estudo de impacto de medida TRIPS-Plus nas compras públicas de medicamentos no Brasil', (CHAVES et al, 2017), o Acordo TRIPS é resultado de uma agenda privada, coordenada por empresas multinacionais e liderada por países desenvolvidos como Estados Unidos, Japão e alguns países europeus. Ainda que com prazos de implementação variados, de acordo com a classificação de desenvolvimento, o Acordo TRIPS estabeleceu para todos os países membros da OMC a obrigatoriedade de reconhecimento da propriedade intelectual em todos os campos tecnológicos (CHAVES et al, 2017).

Desse modo, mesmo com a resistência dos países em desenvolvimento foi estabelecido em 1995 o Agreement on Trade-Related Aspects of Intellectual Property Rights (Acordo TRIPS) da Organização Mundial do Comércio (OMC), que modificou o sistema internacional de propriedade intelectual. Como afirmado acima, o Acordo TRIPS estabeleceu para todos os países membros da OMC a obrigatoriedade de reconhecimento da propriedade intelectual em todos os campos tecnológicos, restringindo tais países a autonomia de adotarem leis e políticas de propriedade intelectual, e encareceu o acesso às inovações tecnológicas. O TRIPS é um acordo de caráter vinculante que implementou padrões mínimos de proteção da propriedade intelectual e estabeleceu mecanismos de sanção aos países que não cumprissem o Acordo.

O direito de propriedade intelectual e a questão das patentes que estão incluídos no acordo TRIPS representam um grande obstáculo para a produção de medicamentos genéricos, posto que criam uma concorrência limitada devido a criação de monopólios. Para as empresas farmacêuticas multinacionais, a proteção da propriedade intelectual, juntamente com as patentes garantem um período de exclusividade no mercado, o que lhes dá o poder de definir os preços dos medicamentos (CHAVES et al, 2017).

Antes do Acordo TRIPS, os países tinham liberdade para definir se iriam ou não patentear certas áreas do conhecimento. Até o final do século $\mathrm{XX}$, os regimes internacionais de propriedade intelectual eram fundamentados no âmbito multilateral pelas Convenções de Paris (1883) e de Berna (1886), que preservavam a base territorial dos direitos de propriedade intelectual (DE MELLO E SOUZA, 2011). Desse modo, essas convenções não criaram leis substantivas, nem obrigavam os países membros a adotar novas leis, o que permitia que cada país membro pudesse adotar as leis e políticas de propriedade intelectual mais adequadas em função das suas vantagens comparativas e dos seus níveis de desenvolvimento tecnológico.

Dessa forma, vários países em desenvolvimento não reconheciam patentes para medicamentos, o que possibilitava uma facilidade para a produção de medicamentos genéricos, que são usados por programas públicos para tratar de forma gratuita doenças como a AIDS e a tuberculose. O acordo TRIPS representou, assim, uma ameaça às políticas de saúde pública, principalmente para os países em desenvolvimento como o Brasil que possui um programa de tratamento para a Síndrome da Imunodeficiência Adquirida (SIDA) - Acquired Immunodeficiency Syndrome (AIDS) - sustentado pelo uso de medicamentos genéricos.

Após pressões dos países em desenvolvimento sobre o acordo TRIPS e suas 
consequências para a saúde pública ficou estabelecido que apesar do TRIPS trazer elementos para a proteção da propriedade intelectual, o acordo também preveria salvaguardas que podem ser adotadas pelos países, a fim de proteger a saúde pública.

As salvaguardas previstas no artigo $31^{\circ}$ do TRIPS são:

I) Períodos de transição: estabeleceu um tempo de transição que corresponde ao nível de desenvolvimento e progresso econômico de cada país com o intuito de possibilitar que os países tenham um tempo hábil para adequar suas legislações de acordo com o TRIPS; II) Licença compulsória: permite a autorização por parte do Estado para explorar invenções patenteadas mediante uma remuneração ao titular da patente, contornando o comportamento anti-competitivo e garantindo a transferência de tecnologia e a disseminação do conhecimento; III) Exceções de uso do governo: concede ao Estado o direito de utilizar a patente sem obter o consentimento do titular para o interesse público, inclusive para fins de saúde pública; IV) Importação paralela: direito de obter produtos patenteados quando estão legalmente disponíveis em um país estrangeiro a um preço mais baixo; V) Padrões de patenteabilidade: de acordo com o TRIPS, a proteção de patentes deve ser concedida a produtos e processos que sejam novos, envolvam uma atividade inventiva e sejam industrialmente aplicáveis. (Artigo $31^{\circ}$ do Acordo TRIPS, 1985, grifo nosso).

No entanto, esses critérios não são definidos e podem ser interpretados e aplicados pelos Estados membros de acordo com suas prioridades nacionais (EL SAID, 2010). Essas flexibilidades estabelecidas no artigo 31 do TRIPS podem ser vistas como uma margem de manobra que possibilita aos países em desenvolvimento garantir que a produção e a disponibilidade de medicamentos nos seus países não sejam prejudicadas pelo acordo TRIPS, essas salvaguardas impedem que a proteção à propriedade intelectual anule o direito à saúde pública (EL SAID, 2010). As salvaguardas permitem que o país possa anular a exclusividade conferida pelo direito de propriedade intelectual e asseguram a entrada de medicamentos genéricos possibilitando, assim, a concorrência para promover a redução dos preços. Esse direito dos países de adotarem tais medidas de proteção da saúde pública foi aprovado no âmbito da OMC em 2001 com a "Declaração de Doha sobre o Acordo TRIPS e a saúde pública".

A Declaração de Doha sobre o Acordo TRIPS e a saúde pública, aconteceu como resultado da adoção de uma Declaração ministerial especial feita pelos países membros da OMC na Conferência Ministerial da OMC em Doha, com o intuito de esclarecer as ambiguidades entre a necessidade de os países aplicarem os princípios de saúde pública e os termos do Acordo TRIPS. Os países em desenvolvimento estavam receosos de que as regras de patentes do TRIPS pudessem restringir o acesso a medicamentos a preços acessíveis para as suas populações, especialmente os medicamentos utilizados no controle de doenças como a AIDS, tuberculose e malária, desse modo, a Declaração de Doha é a solução para essa preocupação visto que, implementou medidas que promovem o acesso a medicamentos a um preço acessível, como afirma o item 4 da Declaração:

4. Concordamos que o Acordo TRIPS não impede e não deve impedir 
os membros de tomar medidas para proteger a saúde pública. Consequentemente, ao mesmo tempo em que reiteramos nosso compromisso com o Acordo TRIPS, afirmamos que o Acordo pode e deve ser interpretado e implementado de forma a apoiar o direito dos Membros da OMC de proteger a saúde pública e, em particular, de promover o acesso a medicamentos para todos. (Declaração de Doha sobre o Acordo TRIPS e a Saúde Pública, 2011).

Desse modo, como exemplifica o item 4 da Declaração de Doha sobre o Acordo TRIPS e a saúde pública, os países podem fazer uso das flexibilidades previstas no artigo 31 do Acordo TRIPS quando julgarem necessário, a fim de proteger a saúde pública e, assim, garantir o acesso de medicamentos a preços acessíveis. Ainda, o item 4 da Declaração ao destacar a promoção do acesso a medicamentos enfatiza a importância dos medicamentos para a promoção da saúde pública.

Ao longo da história, a posição do Brasil acerca do tema de propriedade intelectual e concessão de patentes foi de proteção à saúde pública, visto que até 1996 o Brasil não oferecia proteção de patentes para produtos e processos químico-farmacêuticos, ou seja, possibilitava a cópia e venda de medicamentos de marca no mercado interno por qualquer empresa. Os produtos farmacêuticos, como pontua a pesquisadora De Castro (2018), eram considerados um "bem público" e, desse modo, era permitida a sua cópia o que assegurava aos países em desenvolvimento adotar regimes de propriedade intelectual flexíveis, com o objetivo de garantir a difusão rápida e barata desses medicamentos.

Após a conclusão do Acordo TRIPS, ficou estabelecido que os países em desenvolvimento teriam até 2005 para se adequarem ao novo contexto imposto pelo Acordo TRIPS. No entanto, como destaca a pesquisa "TRIPS e a Saúde Pública: Implicações e Perspectivas" (CORREA, 2018), o Brasil foi alvo de fortes pressões advindas do governo norte americano que estava descontente com a política de propriedade intelectual vigente no país. O Brasil sofreu retaliações comerciais dos Estados Unidos que aplicou sobretaxa a diversos produtos brasileiros, causando graves prejuízos para os exportadores brasileiros de suco de laranja e calçados.

Com isso, a lei $\mathrm{n}^{0}$ 9.279, de 14 de maio de 1996 (nova lei de propriedade intelectual) começou a valer no Brasil em 1996, protegendo os produtos farmacêuticos. Tal lei refletiu as pressões econômicas e políticas dos Estados Unidos contra o Brasil, o que fez o país cumprir as obrigações do Acordo TRIPS antes do prazo estabelecido e introduziu o pedido de depósito retroativo, ou seja, a proteção pipeline.

A proteção pipeline introduzida na legislação brasileira, causou graves impactos no Sistema Único de Saúde visto que, ela vai além das obrigações impostas pelo Acordo TRIPS. A proteção ou patente pipeline é um dispositivo que possibilita a aceitação de depósitos de patentes em campos tecnológicos não reconhecidos anteriormente, e no caso do Brasil está incluso o setor farmacêutico. Conforme explicado pela pesquisadora Chaves $(2020)^{2}$, para uma patente ser concedida ela tem que cumprir com os requisitos

\footnotetext{
2 Entrevista concedida à autora da pesquisa. Gabriela Chaves é doutora em saúde pública e pesquisadora do Núcleo de Assistência Farmacêutica da ENSP/Fiocruz. Trabalhou na Campanha de Acesso a
} 
de patenteabilidade (novidade, atividade inventiva e aplicação industrial) e as patentes pipeline introduzida na legislação brasileira, foram patentes que não tiveram exame quanto aos requisitos de patenteabilidade. A patente pipeline, possibilitou o depósito de pedidos de patentes mesmo fora do período de prioridade e não exigiu o exame técnico do pedido de patente, o que resultou no depósito de 1.182 pedidos de patentes, incluindo medicamentos que são tidos como essenciais ou fundamentais para o combate de problemas de saúde pública no país (CHAVES et al, 2020).

O mecanismo pipeline, possibilitou o depósito de patentes na área farmacêutica entre maio de 1996 e maio de 1997, assegurando proteção com base na análise formal do pedido; na não exploração da invenção no país e na concessão da patente no país de origem; sem passar pelo exame dos requisitos de patenteabilidade de novidade, atividade inventiva e aplicação industrial em âmbito nacional (CORREA, 2018).

Portanto, a pressão imposta pelos Estados Unidos resultou na implementação de uma medida TRIPS-Plus na legislação brasileira sobre propriedade intelectual e impediu que o Brasil utilizasse o período de transição concedido pela Organização Mundial do Comércio para adequar a legislação do país ao Acordo TRIPS.

\section{O Programa de Assistência Farmacêutica do SUS}

De acordo com o artigo $196^{\circ}$ da Constituição Federal de 1988, a saúde é um direito de todos e dever do Estado garantido mediante políticas sociais e econômicas que visem à redução do risco de doença e de outros agravos e ao acesso universal e igualitário, às ações e serviços para sua promoção, proteção e recuperação. Segundo o Ministério da Saúde, o Sistema Único de Saúde é o maior sistema público de saúde do mundo, posto que é o único que atende mais de 100 milhões de habitantes, garantindo o acesso universal e gratuito para todos os cidadãos brasileiros e é financiado com o dinheiro dos impostos arrecadados pelo governo.

A Lei $\mathrm{n}^{\mathrm{o}} 8.080$, de 19 de setembro de 1990 afirma no artigo $2^{\circ}$, inciso $1^{\circ}$, que o dever do Estado de garantir a saúde consiste na formulação e execução de políticas econômicas e sociais que visem à redução de riscos de doenças e de outros agravos e no estabelecimento de condições que assegurem acesso universal e igualitário às ações e aos serviços para a sua promoção, proteção e recuperação. Ainda de acordo com legislação do SUS, está incluída dentro de suas competências a assistência terapêutica integral, incluindo a assistência farmacêutica, ou seja, cabe ao SUS a produção e fornecimento de medicamentos de forma gratuita à população.

A Portaria $n^{\circ}$ 3.916, de 30 de outubro de 1998 estabelece a Política Nacional de Medicamentos, afirmando que a política nacional de medicamentos como parte essencial da Política Nacional de Saúde constitui um dos elementos fundamentais para a efetiva

Medicamentos Essenciais e na Unidade Médica de Médicos Sem Fronteiras no Brasil (2006-2011) e na Associação Brasileira Interdisciplinar de Aids (2006-2008), com atuação no Grupo de Trabalho sobre Propriedade Intelectual da Rede Brasileira pela Integração dos Povos (GTPI/Rebrip). 
implementação de ações capazes de promover a melhoria das condições da assistência à saúde da população. A Lei $\mathrm{n}^{\circ} 8.080 / 90$, em seu artigo $6^{\circ}$, estabelece como campo de atuação do Sistema Único de Saúde (SUS) a "formulação da política de medicamentos (...) de interesse para a saúde (...)" (BRASIL, 1990). O propósito da Política Nacional de Medicamentos é garantir a necessária segurança, eficácia e qualidade dos medicamentos, a promoção do uso racional e o acesso da população àqueles medicamentos considerados essenciais.

Desse modo, em 1999, foi implementado no Brasil a Lei dos Genéricos (Lei ${ }^{\circ}$ 9.787/99) que estabelece um prazo de 20 anos para que os direitos de exclusividade expirem e, com isso, o medicamento possa ser produzido e comercializado de forma genérica. A Lei dos Genéricos tem o intuito de implementar uma política pública que possibilite ampliar o acesso da população aos tratamentos medicamentosos eficazes, seguros e mais baratos no Brasil.

A produção de medicamentos genéricos no Brasil possibilita que a assistência farmacêutica do Sistema Único de Saúde seja universal, visto que os medicamentos genéricos conseguem ser $73 \%$ mais baratos do que os medicamentos de referência. De acordo com a Agência Nacional de Vigilância Sanitária (Anvisa), o medicamento genérico é aquele que possui o mesmo princípio ativo, forma farmacêutica, via de administração, posologia e indicação terapêutica, preventiva ou diagnóstica do medicamento de referência e, por isso, é intercambiável com o medicamento de referência. A única coisa que o difere de um medicamento genérico do medicamento de referência é a falta do nome comercial, ou seja, o medicamento genérico é uma cópia do medicamento original que teve a sua patente expirada, possibilitando a fabricação da versão genérica. Desse modo, como o medicamento genérico não possui uma patente e, portanto, não é exclusivo, o que de acordo com a Associação Brasileira das Indústrias de Medicamentos Genéricos (Pró Genéricos) possibilita que ele seja, no mínimo 35\% mais barato do que os medicamentos de referência que são patenteados.

Ainda a empresa responsável pela produção de medicamentos genéricos deve possuir um certificado de boas práticas de fabricação e controle de qualidade que é concedido após uma inspeção realizada pela Anvisa, a qual verifica a garantia da qualidade dos medicamentos. De acordo com a OMS é preciso também comprovar a eficácia terapêutica, a segurança e a intercambialidade ${ }^{3}$ dos genéricos através da realização dos ensaios de biodisponibilidade (teste de quantidade e velocidade de absorção do princípio ativo do medicamento para a corrente sanguínea, ou seja, quando dois medicamentos apresentam a mesma biodisponibilidade no organismo, sua eficácia clínica é considerada compatível) e bioequivalência (consiste na demonstração de que o medicamento genérico e seu respectivo medicamento de referência apresentam a mesma biodisponibilidade no organismo, ou seja, assegura que o medicamento genérico apresenta a mesma eficácia clínica e a mesma segurança em relação ao medicamento de referência).

\footnotetext{
3 A intercambialidade se trata de uma característica que indica as possibilidades de substituição de medicamentos considerados equivalentes (ANVISA, 2021).
} 
O Sistema Único de Saúde é o principal comprador de medicamentos no Brasil, posto que na Constituição de 1988 foi estabelecido o acesso universal à saúde e, posteriormente, foi garantido o direito à assistência farmacêutica através do SUS com a Política Nacional de Medicamentos que entende o medicamento como um insumo essencial para a promoção e recuperação da saúde. Além disso, é preciso pontuar que no Brasil alguns medicamentos como os antirretrovirais e os destinados para o tratamento da tuberculose não são vendidos nas farmácias privadas. Esses medicamentos são distribuídos apenas pelo Sistema Único de Saúde.

O Brasil foi o primeiro país em desenvolvimento a implementar um programa de distribuição universal em larga escala de antirretrovirais, tal programa serviu de modelo para alguns países em desenvolvimento (CORREA, 2018). A implementação dessa política de acesso universal e gratuito aos pacientes de AIDS a partir de 1996 enfrentou as complicações do fortalecimento da propriedade intelectual, posto que o acordo TRIPS encarece o acesso às inovações tecnológicas e, desse modo, ameaça as políticas de saúde pública do SUS.

A Lei dos Genéricos foi, portanto, essencial para estimular a produção local de medicamentos genéricos e possibilitar o tratamento gratuito e universal aos pacientes de AIDS. De acordo com o pesquisador De Mello E Souza (2011), a sustentabilidade do programa brasileiro de tratamento da Síndrome da Imunodeficiência Adquirida tem sido garantida pela produção doméstica de medicamentos genéricos, que possibilita a substituição da importação de antirretrovirais caros não patenteados no Brasil e a obtenção de descontos das multinacionais farmacêuticas para os antirretrovirais patenteados no país devido a ameaça do governo brasileiro a essas empresas do uso de licenciamento compulsório.

O principal laboratório público brasileiro capacitado pelo governo para realizar a fabricação e o fornecimento de versões genéricas dos medicamentos antirretrovirais é o Instituto de Tecnologia em Fármacos (Farmanguinhos). Desde que o Ministério da Saúde começou a substituir as importações de antirretrovirais caros por equivalentes genéricos produzidos no Brasil, os preços desses medicamentos tiveram uma queda média de quase 81\% até 2001 (DE MELLO E SOUZA, 2011). Desse modo, a Farmanguinhos e outros laboratórios brasileiros foram capazes de baratear esses medicamentos estabelecendo margens de lucros inferiores às das multinacionais farmacêuticas e quebrando os monopólios.

É importante ressaltar que, após a retificação do Acordo TRIPS, os medicamentos patenteados utilizados no tratamento do HIV/AIDS foram comercializados a preços inacessíveis e comprometeram a capacidade dos países de ofertarem tratamento à sua população. A garantia da assistência farmacêutica no Sistema Único de Saúde representa avanços importantes na ampliação do acesso a medicamentos pela população brasileira. Entretanto, tem enfrentado desafios significativos para a sustentabilidade desse acesso, pois a incorporação de novas tecnologias em situação de monopólio aumentou os gastos do governo de R\$ 8,5 bilhões em 2008 para R\$ 14,8 bilhões em 2015 (CHAVES et al, 2017).

Ademais, um dos medicamentos utilizados no coquetel contra a AIDS, o Efavirenz, foi motivo de desavenças entre o governo brasileiro e o laboratório norte 
americano Merck em 2007. Após o Brasil fazer uso da licença compulsória fundamentada no interesse público de assegurar o programa brasileiro de tratamento do HIV/AIDS a fabricante do medicamento, a Merck Sharp e Dohme acusou o Ministério da Saúde de não querer negociar preços. Essa situação é um exemplo nítido sobre o conflito de interesses entre a questão de patentes e as flexibilidades do Acordo TRIPS.

É necessário destacar que, conforme aponta o artigo " O Instituto de Patentes Pipeline e o Acesso a Medicamentos: Aspectos Econômicos e Jurídicos Deletérios à Economia da Saúde" (CHAVES et al, 2020), dentre os 19 antirretrovirais fornecidos pelo Ministério da Saúde, apenas 8 não são protegidos por patentes e podem ser produzidos pelos laboratórios nacionais públicos e privados. Os demais antirretrovirais, são patenteados ou estão sob proteção no Brasil e, desse modo, são importados de empresas farmacêuticas transnacionais o que encarece os gastos do Ministério da Saúde.

\section{As Medidas TRIPS PLUS e o Acordo entre o MERCOSUL e a União Europeia}

As medidas TRIPS Plus são dispositivos que tornam a legislação mais restritiva do que o estabelecido pelo Acordo TRIPS. Tais medidas anulam em diversos níveis as salvaguardas previstas no artigo 31 do TRIPS e dificultam a produção de medicamentos genéricos. As medidas TRIPS Plus favorecem o direito do titular da patente e restringe o uso das salvaguardas, desse modo, o equilíbrio que foi buscado com a adoção das flexibilidades do TRIPS fica comprometido (CHAVES, 2020).

Os dispositivos ou cláusulas TRIPS Plus vão além do Acordo TRIPS visto que, fortalecem o poder conferido pela propriedade intelectual e restringe o espaço para a adoção de medidas que diminuem os efeitos causados pelo abuso do poder de monopólio conferido pela propriedade intelectual. Geralmente essas medidas são encontradas nos acordos bilaterais de livre comércio, como o Acordo entre o Mercosul e a União Europeia.

Os dispositivos ou cláusulas TRIPS-Plus fortalecem o poder conferido pelo direito de propriedade intelectual restringindo o espaço de ação de medidas que diminuem os efeitos decorrentes do abuso de poder de monopólio conferido pela propriedade intelectual. A proteção da propriedade intelectual é crucial para o setor farmacêutico, visto que é um instrumento importante para as suas estratégias comerciais e para a inovação, pois as patentes garantem às empresas um período de exclusividade no mercado para seus produtos, excluindo, assim, a participação de terceiros. Desse modo, a patente confere o poder de definição dos preços para essas empresas que, em tese, possibilitam a recuperação dos custos de pesquisa e desenvolvimento (P \& D) (CHAVES et al, 2017).

As medidas TRIPS Plus relacionadas à saúde incluem a eliminação e redução dos períodos de transição, proteção de exclusividade de dados, extensão de termos de proteção de patente, restrições à importação paralela, patenteabilidade de novo uso de substâncias médicas conhecidas, restrições ao licenciamento compulsório, patenteamento de formas de vida e limitações aos critérios de patenteabilidade, o que resulta em um impacto direto na saúde pública, na produção farmacêutica, na disponibilidade e preços dos medicamentos. (El Said, 2010)

No capítulo de propriedade intelectual do acordo de livre comércio entre 
o Mercosul e a União Europeia, é possível identificar no item 6 do artigo X.3 uma abertura para a implementação de medidas TRIPS Plus pela União Europeia:

\begin{abstract}
6. Nenhuma Parte será obrigada a implementar em sua legislação uma proteção mais ampla do que a exigida por este Capítulo. Este Capítulo não impede que as Partes apliquem disposições de direito interno que introduzam padrões mais elevados para a proteção e ampliação da propriedade intelectual, desde que não violem as disposições deste Capítulo. (capítulo de propriedade intelectual do acordo de livre comércio MERCOSUL- União Europeia, item 6 do artigo X.3, tradução nossa).
\end{abstract}

De acordo com a conclusão do estudo "Tratado de livre comércio União Europeia Mercosul: Estudo de impacto de medida TRIPS-Plus nas compras públicas de medicamentos no Brasil" (CHAVES et al, 2017), se for implementada no acordo a medida TRIPS Plus proposta anteriormente pela União Europeia, que estende a vigência da proteção patentária dos medicamentos antirretrovirais, o gasto adicional para o Ministério da Saúde seria de aproximadamente R \$ 1,22 bilhões para apenas 6 medicamentos. Desse modo, a implementação de medidas TRIPS Plus no Acordo de Livre Comércio entre os dois blocos teria um impacto negativo no SUS, em especial nas políticas públicas de acesso a medicamentos no Brasil, tal como o Programa Farmácia Popular.

Chaves (2020) argumenta ainda que se as medidas TRIPS PLUS sugeridas pela União Europeia entrarem em vigor, elas prejudicariam a produção e o fornecimento de medicamentos genéricos no Brasil, visto que qualquer medida TRIPS PLUS que garanta o monopólio da patente impede que terceiros explorem a invenção. Portanto, tal monopólio afeta diretamente qualquer esforço de produção pública de medicamentos genéricos.

Ademais, a pesquisa "Empty Gestures: The EU'S Commitments to Safeguard Access to Medicines" (HAI \& MSF, 2015) aponta um padrão de inclusão de regras de propriedade intelectual TRIPS PLUS para os produtos farmacêuticos nas negociações dos acordos comerciais realizados pela União Europeia. Todos os acordos de livre comércio da União Europeia incluem uma referência padrão que confirma sua adesão à declaração de Doha, no entanto as disposições substantivas do acordo incluem disposições TRIPS PLUS. Um exemplo disso é o Acordo de Livre Comércio entre a União Europeia e o CARIFORUM, no qual o capítulo de propriedade intelectual do acordo afirmar sua posição de defesa da saúde pública, mas deixa uma abertura para a implementação de medidas TRIPS PLUS nos países membros do CARIFORUM.

A parte CE e os Estados do CARIFORUM signatários podem, mas não são obrigados a implementar na sua legislação uma proteção mais ampla do que a exigida pela presente secção, desde que tal proteção não infrinja as disposições desta secção. (Artigo $139^{\circ}$, item $4^{\circ}$ do Acordo de Livre Comércio entre a União Europeia e o CARIFORUM, tradução nossa).

A parte CE e os Estados do CARIFORUM comprometem-se a avançar para um nível harmonizado de proteção da propriedade intelectual nas 
respectivas regiões. (Artigo $141^{\circ}$, item $2^{\circ}$ do Acordo de Livre Comércio entre a União Europeia e o Cariforum, tradução nossa).

Desse modo, fica evidente que a União Europeia ao deixar uma interpretação em aberta sobre o Acordo TRIPS não respeita as salvaguardas garantidas pela Declaração de Doha, visto que a União Europeia cria obstáculos para impedir que os países tomem medidas para proteger a saúde pública. O mesmo ocorre no Acordo entre o MERCOSUL e a União Europeia visto que, como citado anteriormente, o item 6 do artigo X.3 do capítulo de propriedade intelectual do Acordo de Livre Comércio entre os dois blocos deixa uma abertura para a implementação de medidas TRIPS PLUS pela União Europeia.

É importante destacar que no acordo preliminar havia medidas TRIPS PLUS explícitas sugeridas pela União Europeia conforme identificado pelo estudo "Tratado de Livre Comércio União Europeia Mercosul: Estudo de impacto de medida TRIPS-Plus nas compras públicas de medicamentos no Brasil" (CHAVES et al, 2017). O estudo identificou três medidas TRIPS PLUS que afetariam negativamente a saúde pública brasileira. São elas:

I) Restrição da Importação Paralela, a importação paralela é uma das salvaguardas previstas no TRIPS, desse modo, a restrição dessa salvaguarda aconteceria por meio da adoção do regime nacional ou internacional de exaustão de direitos por ambos os blocos; II) Adoção de exclusividade de dados de testes: as partes não permitirão que qualquer outro fabricante do mesmo produto ou produto similar obtenha aprovação para comercialização com base na aprovação de comercialização concedida ao fabricante que tenha fornecido os resultados dos ensaios pré-clínicos ou clínicos, por um período de [...] anos (o número de anos não está determinado na proposta); e III) Extensão do tempo de vigência da patente: os países teriam que estender o prazo de validade de uma patente de medicamento que tenha sido submetido a um procedimento de autorização administrativa para a sua comercialização, o que dificultaria a produção genérica deste medicamento (CHAVES et al, 2017).

Se tais medidas forem implementadas, tanto o Programa Nacional de Medicamentos do SUS quanto a produção de medicamentos genéricos no Brasil serão negativamente afetados, pois caso essas propostas da União Europeia para o capítulo de propriedade intelectual do acordo entre os dois blocos forem implementadas, o Brasil terá que desembolsar um valor adicional de até R\$ 1,9 bilhão por ano apenas para realizar a compra desses medicamentos (CHAVES, 2017).

A proposta de extensão da vigência das patentes de medicamentos feita pela União Europeia no Acordo inicial causaria um gasto adicional para o Ministério da Saúde de US\$ 444.081.767,74 para apenas 6 medicamentos (Darunavir; Etravirine; Raltegravir; Sofosbuvir; Daclatasvir; e Dasatinib) representando, assim, 8,24\% das despesas do Ministério da Saúde em 2015 (CHAVES et al, 2017).

É importante ressaltar que durante essa simulação a taxa de câmbio considerada era de US\$1,00 $=\mathrm{R} \$ 2,75$. Portanto, com a alta do dólar esse valor sobe ainda mais tornando extremamente difícil a manutenção do acesso universal a esses 
medicamentos pelo SUS colocando, assim, em risco a saúde pública brasileira.

\section{Conclusão}

O Acordo TRIPS estabeleceu um padrão mínimo de proteção da propriedade intelectual modificando de maneira significativa os patamares de proteção da propriedade intelectual previstos nos países em desenvolvimento. Por ser considerado um padrão mínimo, o TRIPS possibilitou que medidas mais fortes fossem adotadas em um contexto de maior assimetria entre os países envolvidos em acordos bilaterais, isso fica nítido quando olhamos para o acordo comercial entre a União Europeia e o CARIFORUM.

Ao restringir e impedir o uso das flexibilidades do artigo 31 do TRIPS, as medidas TRIPS Plus ameaçam a política de assistência farmacêutica do Sistema Único de Saúde, visto que por estabelecerem medidas mais fortes de proteção da propriedade intelectual, como o aumento da proteção patentária dos medicamentos e não possibilitarem o uso das salvaguardas do TRIPS, as medidas TRIPS Plus afetariam diretamente a produção de medicamentos genéricos no Brasil e, consequentemente, aumentariam os gastos do Ministério da Saúde colocando em risco a assistência farmacêutica universal do SUS.

A tendência é que haja um aumento na demanda por medicamentos no SUS visto que, a população tende a crescer e, desse modo, também aumenta os gastos do Ministério da Saúde com a compra de medicamentos que estão sob proteção de patentes farmacêuticas. Além disso, é preciso considerar a alta do dólar visto que, com a atual crise econômica e política no Brasil há uma alta considerável do dólar o que encarece a importação dos medicamentos essenciais para a promoção da saúde dos brasileiros.

Como as medidas TRIPS Plus restringem a produção de medicamentos genéricos, o Ministério da Saúde consequentemente teria que aumentar a importação de diversos medicamentos, o que levará ao aumento dos gastos do SUS com medicamentos colocando em risco, a assistência farmacêutica, pois com o aumento da demanda por medicamentos juntamente com o aumento dos gastos do Ministério da Saúde na compra desses medicamentos, a manutenção da Política Nacional de Medicamentos ficaria insustentável.

Portanto, as medidas TRIPS Plus sugeridas pela União Europeia no texto preliminar do Acordo de Livre Comércio com o Mercosul ferem diretamente o direito à saúde assegurado pelo Artigo 196 da Constituição Federal de 1988. Desse modo, qualquer tentativa de implementação de medidas TRIPS Plus nos países do Mercosul através do Acordo de Livre Comércio com a União EuropEia terá graves consequências para a saúde pública de todos os países membros do Mercosul, em especial para o país que foi o foco deste artigo, o Brasil. Já que essas medidas causariam um impacto direto ao direito de acesso à medicamentos no país.

A abertura para uma possível elevação do direito de propriedade intelectual sugerida pela União Europeia, deixada no texto do Acordo no item 6 do artigo X.3 do capítulo de propriedade intelectual, representa um risco para uma possível jogada por parte da União Europeia. Em outras palavras, mesmo com a retirada das medidas TRIPS Plus do texto final do Acordo, foi deixada uma abertura para uma possível implementação dessas medidas no futuro. 
O histórico da União Europeia de exigir, mesmo que de forma sutil, um aumento na proteção da propriedade intelectual nos seus acordos bilaterais representa um risco futuro para a saúde pública brasileira.

\section{Referências Bibliográficas}

AGÊNCIA NACIONAL DE VIGILÂNCIA SANITÁRIA (Anvisa). Publicada Lista de Medicamentos Similares Intercambiáveis. Anvisa website, 24 fev. 2021. Disponível em: $<$ https://www.gov.br/anvisa/pt$\mathrm{br} /$ assuntos/noticias-anvisa/anos-anteriores/publicada-lista-de-medicamentos-similares-intercambiaveis $>$ Acesso em: 29 nov. 2021.

_. Conceitos e Definições. Anvisa website, s.d. Disponível em: $<$ https://www.gov.br/anvisa/pt$\mathrm{br} / \mathrm{acessoainformacao/perguntasfrequentes/medicamentos/conceitos-e-definicoes}>$. Acesso em: 01 set. 2020 .

AOUN, Alejandra; BARRENECHEA, Alejo; BLASETTI, Roxana et al. Estudio Preliminar Del Capítulo Sobre Propiedad Intelectual Del Acuerdo Mercosur- UE. Genebra: South Centre, 2020. Disponível em: <https://www.southcentre.int/wp-content/uploads/2020/05/RP-110.pdf > Acesso em: 15 ago. 2020.

ASSOCIAÇÃO BRASILEIRA DAS INDÚSTRIAS DE MEDICAMNTOS E BIOSSIMILARES (Progenéricos). O que são os medicamentos genéricos? Progenéricos website, s.d. Disponível em: $<$ https://www.progenericos.org.br/genericos> Acesso em: 28 ago. 2020.

BERMUDEZ, Jorge. Medicamentos Genéricos: uma alternativa para o mercado brasileiro. Cadernos de Saúde Pública, v. 10, n.3, set. 1994. Disponível em:

$<$ https://www.scielo.br/scielo.php?script=sci_arttext\& pid=S0102-311X1994000300016>. Acesso em: 08 ago. 2020 .

BRASIL. Decreto n 9.289, de 21 de fevereiro de 2018. Brasília: Governo Federal, Diário Ofícial, 2018. Disponível em <http://www.planalto.gov.br/ccivil_03/_ato2015-2018/2018/decreto/D9289.htm> Acesso em: 28 ago. 2020.

. Lei n 9.787, de 10 de fevereiro de 1999. Brasília: Governo Federal, Diário Ofícial, 1999. Disponível em <http://www.planalto.gov.br/ccivil_03/leis/19787.htm> Acesso em: 4 set. 2020.

Portaria n³.916, de 30 de outubro de 1998. Brasília: Governo Federal, Diário Oficial, 1998. Disponível em <http://bvsms.saude.gov.br/bvs/saudelegis/gm/1998/prt3916_30_10_1998.html> Acesso em: 3 set. 2020.

Lei no 9.279, de 14 de maio de 1996. Brasília: Governo Federal, Diário Oficial, 1996. Disponível em $<$ http://www.planalto.gov.br/ccivil_03/leis/19787.htm> Acesso em: 3 set. 2020.

Lei n 8.080, de 19 de setembro de 1990. Brasília: Governo Federal, Diário Oficial, 1990. Disponível em <http://www.planalto.gov.br/ccivil_03/leis/18080.htm> Acesso em: 17 ago. 2020.

CHAVES, Gabriela. Entrevista sobre o capítulo de propriedade intelectual do texto final do Acordo de Livre Comércio entre a União Europeia e o Mercosul. 25 de novembro de 2020. Zoom. 
Entrevista realizada com autora.

_. Mercosur-EU Free Trade Agreement: Impact analysis of TRIPS-Plus measures proposed by the EU on public purchases and domestic production of HIV and Hepatitis C medicines in Brazil. Rio de Janeiro: Fiocruz, 2017.

CHAVES, Gabriela; FOGAÇA VIEIRA, Marcela; GASPAR BRITTO, Walter; PRABHALA, Achal. Tratado de Livre Comércio União Europeia- Mercosul: Estudo de impacto de medida TRIPS-Plus nas compras públicas de medicamentos no Brasil. Rio de Janeiro: FIOCRUZ, 2017.

CHAVES, Gabriela; FOGAÇA, Marcela; VIEIRA, Renata. Access to Medicines and Intellectual Property in Brazil: Reflections and Strategies of Civil Society. Sur: Revista Internacional de Direitos Humanos, v.5, n.8, 2008, p.170-198.

CONFEDERAÇÃO NACIONAL DA INDÚSTRIA. Acordo Mercosul e União Europeia: análise do capítulo sobre propriedade intelectual. Brasília: CNI, 2019.

CORREA, Carlos. TRIPS Agreement and access to Drugs in Developing Countries. In: DE CASTRO, Elza. O Acordo TRIPS e a Saúde Pública: Implicações e perspectivas. Brasília: Fundação Alexandre de Gusmão, 2018.

. Patentes e Medicamentos Genéricos. Le Monde Diplomatique, $1^{\circ}$ jan. 2008. Disponível em: $<$ https://diplomatique.org.br/patentes-e-medicamentos-genericos/>. Acesso em: 29 ago. 2020.

DE MELlO E SOUZA, André. O Acordo sobre os Aspectos dos Direitos de Propriedade Intelectual Relacionados ao Comércio (TRIPS): Implicações e possibilidades para a saúde pública no Brasil. Rio de Janeiro: IPEA, 2011.

EIFLER, Fernando. Descrição dos Sistemas de Saúde Pública de Argentina, Brasil e Uruguai. Medium, 2016. Disponível em: <https://medium.com/@fernandoeifler/descrição-dos-sistemas-de-saúde-públicade-argentina-brasil-e-uruguai-4b2f294dca05>. Acesso em: 17 ago. 2020.

ECONOMIC PARTNERSHIP AGREEMENT between the CARIFORUM States, of the one part, and the European Community and its Member States, of the other part. Disponível em:

$<$ http://www.sice.oas.org/Trade/CAR_EU_EPA_e/CAR_EU_e.asp>. Acesso em: 03 out. 2020.

EL SAID, Mohammed. Public Health Related TRIPS Plus Provisions in Bilateral Trade Agreements: A Policy Guide for negotiators and implementers in the WHO Eastern Mediterranean Region. World Health Organization and International Centre for Trade and Sustainable Development, 2010.

FIOCRUZ. União Europeia propõe medidas que podem aumentar os gastos públicos do Brasil com medicamentos no Tratado de Livre Comércio. FIOCRUZ, 2017. Disponível em:

$<$ https://www.cee.fiocruz.br/?q=node/656> Acesso em: 29 ago. 2020.

_. O que é um genérico? Fiocruz website, s.d. Disponível em: <https://www.far.fiocruz.br/categoria-domedicamento/o-que-e-um-generico/?print=print> Acesso em: 28 ago. 2020.

HEALTH ACTION INTERNATIONAL (HAI); MÉDECINS SANS FRONTIÈRES (MSF). Empty Gestures: The EU'S Commitments to Safeguard Access to Medicines. Review of the European Union's Trade \& Investment Policy, set. 2015. Disponível em:

$<$ https://rade.ec.europa.eu/doclib/docs/2015/october/tradoc_153873.pdf $>$ Acesso em: 05 set. 2020.

HASENCLEVER, Lia; LOPES, Rodrigo; CHAVES, Gabriela; REIS, Renata; VIEIRA, Marcela. O instituto de patentes pipeline e o acesso a medicamentos: Aspectos econômicos e jurídicos 
deletérios à economia da saúde. Revista de Direito Sanitário, v. 11, n.2, jul.-out. 2010, p.164-188. Disponível em: <https://www.revistas.usp.br/rdisan/article/view/13212/15024>. Acesso em: 15 ago. 2020.

MIRANDA, Pedro Henrique Marques Villardi. Perguntas e Respostas sobre Patentes Pipeline: como afetam sua saúde? Rio de Janeiro: ABIA, 2009.

MINISTÉRIO DAS RELAÇÕES EXTERIORES. Texto do Acordo Mercosul-União Europeia. Ministério das Relações Exteriores, 2019. Disponível em: <http://www.itamaraty.gov.br/pt-BR/notas-aimprensa/20626-texto-do-acordo-Mercosul-uniao-europeia > Acesso em: 19 ago. 2020.

OBSERVATÓRIO IBERO-AMERICANO DE POLÍTICAS PÚBLICAS EM SAÚDE BUCAL. Sistema de Saúde- Argentina. Observatório Ibero-Americano... website, s.d. Disponível em:

$<\mathrm{https}$ ://sites.usp.br/iberoamericanoralhealth/perfil/argentina/sistema-de-saude-argentina/ >. Acesso em: 18 ago. 2020.

_. Sistema de Saúde- Paraguai. Observatório Ibero-Americano... website, s.d. Disponível em: $\overline{<}$ htps://sites.usp.br/iberoamericanoralhealth/perfil/paraguai/sistema-de-saude-paraguai/> Acesso em: 18 ago. 2020..

ORGANIZAÇÃO MUNDIAL DO COMÉRCIO. Declaração de Doha: Acordo TRIPS e a Saúde Pública, 20 nov. 2001. Disponível em:

$<$ https://www.wto.org/english/thewto_e/minist_e/min01_e/mindecl_trips_e.htm>. Acesso em: 22 ago. 2020.

PORTAL EDUCAÇÃO. SUS: Princípios e diretrizes. Portal Educação website, s.d. Disponível em: $<$ https://siteantigo.portaleducacao.com.br/conteudo/artigos/medicina/sus-principios-e-diretrizes/38572> Acesso em: 20 ago. 2020.

VENTURA, Deisy. Saúde Pública e Integração Regional: tensões entre o direito à saúde e o comércio internacional. In: VON BOGDANDY, Armin; PIOVESAN, Flávia; ANTONIAZZI, Mariela Morales (Orgs.). Direitos Humanos, Democracia e Integração Jurídica na América do Sul. Rio de Janeiro: Max Planck Institute/Lumen Juris/PUC-SP, 2011, v. 1, p. 449-472.

VIEIRA, Fabiola Sulpino. Evolução do Gasto com Medicamentos do Sistema Único de Saúde no Período de 2010 a 2016. Rio de Janeiro: Instituto de Pesquisa Econômica Aplicada, 2018. 